\title{
Gestão do Conhecimento no setor da saúde: mapeamento de ativos de conhecimento com o CommonKADS
}

\section{Knowledge Management in Health Care: knowledge assets mapping with CommonKADS}

\author{
Mariângela Poleza Doutoranda em Engenharia e Gestão do Conhecimento. Universidade Federal de Santa Catarina \\ (UFSC) - Brasil. mapoleza@outlook.com \\ Guillermo Antonio Dávila Doutor em Engenharia e Gestão do Conhecimento. Universidade Federal de Santa Catarina \\ (UFSC) - Brasil. davila.guillermo@gmail.com \\ Divino Ignácio Ribeiro Junior Doutor em Engenharia e Gestão do Conhecimento. Universidade do Estado de Santa Catarina \\ (UFSC) - Brasil. divinoirj@gmail.com
}

\begin{abstract}
RESUMO
Este estudo teve como objetivo compreender como os ativos de conhecimento estão inseridos no processo de atendimento ao paciente em uma organização do setor da saúde. Para tanto, realizou-se um estudo de caso em uma organização de saúde, em que os dados foram coletados por meio de observação e entrevista. A metodologia CommonKADS foi utilizada para analisar e organizar os resultados. Os resultados evidenciaram que dos seis ativos de conhecimento identificados, o ativo de conhecimento referente ao grupo de estudos não está na forma, lugar, tempo e qualidade corretos. Isto influencia diretamente no processo de criação do conhecimento, que fica prejudicado, uma vez que apenas a socialização acontece de forma adequada. Assim, para este caso sugeriu-se a utilização de agentes computacionais para que todas as etapas de criação do conhecimento possam acontecer, sendo que a viabilidade desta proposta foi demonstrada no estudo. Ademais, os resultados demonstraram que a cultura organizacional, orientada pelo líder, favorece o compartilhamento do conhecimento na organização estudada, bem como a prestação de um atendimento de qualidade aos pacientes. Nossos resultados contribuem com a visão da firma baseada em conhecimento, com pesquisas empíricas relacionadas à Gestão do Conhecimento em organizações de saúde de países emergentes e exemplifica a aplicação do CommonKADS. Futuras pesquisas podem analisar empresas de outros setores intensivos em conhecimento, utilizando métodos que permitam entender aspectos dinâmicos da criação do conhecimento nas organizações.
\end{abstract}

Palavras-chave: Gestão do conhecimento. Organização de saúde. CommonKADS. Engenharia do conhecimento. Estudo de caso.

\begin{abstract}
This study aimed to understand how knowledge assets are embedded in the patient care process in a healthcare organization. We conducted a case study in an organization of the health sector and we used observation and interviews for collecting data. We followed guidelines from CommonKADS for analyzing and organizing the results. The results showed that of the six knowledge assets identified, the knowledge asset related to the study group is not in the correct form, place, time and quality. This directly influences the process of knowledge creation, which is impaired, since only socialization happens properly. Thus, for this case we suggested the use of computational agents so that all stages of knowledge creation can happen, and the feasibility of this proposal was demonstrated in the study. Moreover, the results showed that the organizational culture, guided by the leader, favors the sharing of knowledge in the studied organization, as well as the providing of quality care to patients. Our findings contribute to the knowledge-based view, with empirical research related to Knowledge Management in health organizations in emerging countries and exemplify the application of CommonKADS. Future research may explore cases in other knowledge-intensive sectors, and may use methods for analyzing the dynamic aspects of organizational knowledge creation.
\end{abstract}

Keywords: Knowledge management. Health organization. CommonKADS. Knowledge Engineering. Case study. 


\section{INTRODUÇÃO}

O conhecimento tem papel fundamental na economia atual, designada como economia do conhecimento ou economia pós-industrial (Kabir, 2019). Isso se deve à relevância do conhecimento, como um recurso organizacional, que favorece a inovação e a vantagem competitiva sustentável (Grant, 1996; Nonaka \& Takeuchi, 1997; Kaplan \& Norton, 2004). Portanto, é importante que as organizações façam a gestão eficiente do conhecimento (Gonzalez \& Martins, 2017).

A Gestão do Conhecimento (GC) é um processo sistemático e coletivo que promove o tratamento e uso do conhecimento organizacional para atingir os objetivos estratégicos e operacionais (North \& Kumta, 2018). Ademais, é reconhecido que uma área de estudos, que trabalha de maneira complementar e interdisciplinar com a GC, é a Engenharia do Conhecimento (EC). Esta oferece técnicas, ferramentas e métodos para o desenvolvimento de sistemas computacionais para apoiar o processo de GC (Schreiber et al., 2000).

A importância da GC se estende, também, às organizações de saúde, visto que há uma preocupação dos profissionais da área em preservar o conhecimento organizacional, dadas as demandas de saúde da população e do sistema vigente (Rocha et al., 2012; Cicone, Costa, Massuda, Vermelho \& Gimenes, 2015), bem como o reconhecimento da influência positiva no desempenho organizacional, tomada de decisão, competitividade, diminuição de custos, redução de erros clínicos e qualidade do atendimento (El Morr \& Subercaze, 2010; Cruz \& Ferreira, 2016).

Entretanto, as organizações de saúde têm muitas particularidades e os profissionais que nelas atuam enfrentam diferentes desafios, tornam-se ainda maiores devido à falta de GC, na realização de suas atividades diárias e tomadas de decisão (Cegarra-Navarro \& Cepeda-Carrión, 2010; El Morr \& Subercaze, 2010; Liu, Cheng, Chao, \& Tseng, 2012). Neste sentido, os problemas, em geral, referem-se à quantidade de conhecimento organizacional criado diariamente, uma vez que falta estruturação dos processos organizacionais e compartilhamento de experiências; faltam sistemas de apoio à decisão, com protocolos de prescrições e tratamentos, para evitar erros médicos; e existe a necessidade de organizar o conhecimento disperso a fim de torná-lo acessível (El Morr \& Subercaze, 2010).

Ademais, reconhece-se que as organizações de saúde são intensivas em conhecimento (Cruz \& Ferreira, 2016), em que o compartilhamento de conhecimentos tácito e explícito são essenciais para a prestação de serviços de saúde com qualidade (Assem \& Pabbi, 2016). Estas caracterizam-se pela intensidade de uso de conhecimento em suas atividades (Starbuck, 1992) e nelas prevalece o trabalho de natureza intelectual (Alvesson, 2004).

Sendo o conhecimento, o principal recurso para a competitividade nestes tipos de organizações, é possível prever que o mesmo merece uma maior atenção por parte dos gestores pois, um adequado gerenciamento dele, terá um impacto maior nos resultados destas organizações como demostrado em estudos em países desenvolvidos (Kianto \& Andreeva, 2014; Inkinen, Kianto, \& Vanhala, 2015) e emergentes, como o Brasil (Davila, North, \& Varvakis, 2019; Davila, Andreeva, \& Varvakis, 2019). Como todo recurso, o conhecimento envolvido nos processos-chave das organizações de saúde, para ser gerenciado, precisa, primeiro, ser adequadamente mapeado e caracterizado.

Diante deste contexto, pergunta-se: Quais são os ativos de conhecimento presentes em uma organização de saúde? Quais são os atores que geram esse conhecimento? Em quais tarefas organizacionais eles estão inseridos? Como esse conhecimento é gerenciado durante estas tarefas? Para responder estas perguntas, em um estudo de caso, utilizou-se o CommonKADS, como metodologia de Engenharia do Conhecimento, para compreender como os ativos de conhecimento estão inseridos no processo de atendimento ao paciente em uma organização do setor da saúde.

Esta pesquisa contribui com a visão baseada no conhecimento (do inglês knowledge-based view KBV) (Grant, 1996), pois avança nos estudos a respeito do entendimento da integração do conhecimento especializado individual na produção de bens e serviços, em organizações intensivas em conhecimento, em países emergentes. No setor da saúde, contribui para o avanço das pesquisas relacionadas à Gestão do Conhecimento, especificamente no que diz respeito a identificar o conhecimento existente nas organizações e definir estratégias para gerenciá-lo; minimizar o gap relacionado à carência de soluções para problemas 
relacionados com a falta de gerenciamento do conhecimento (Rocha et al., 2012); e contribuir com a redução da escassez de estudos empíricos sobre GC neste setor (Cicone et al., 2015; Cruz \& Ferreira, 2016).

\section{FUNDAMENTAÇÃO TEÓRICA}

Para contextualização e compreensão acerca dos temas relacionados ao estudo, nesta seção apresenta-se a conceituação de conhecimento e Gestão do Conhecimento; metodologias de Engenharia do Conhecimento; e CommonKADS.

\subsection{Conhecimento e Gestão do Conhecimento}

Conhecimento é um conceito que pode ser definido no domínio subjetivo e objetivo. No domínio subjetivo o conhecimento é interno ao indivíduo (como um pensamento), entendido como conhecimento individual; no domínio objetivo o conhecimento é externo ao indivíduo (por exemplo, nos sistemas e procedimentos organizacionais), entendido como conhecimento coletivo. Neste sentido, em concordância com os objetivos da pesquisa e o método utilizado, neste estudo o conhecimento é um pensamento na mente do indivíduo, caracterizado pelas crenças individuais e justificáveis do que é verdadeiro, que pode ser empírico ou não empírico, como por exemplo, o conhecimento matemático, religioso ou filosófico (Zins, 2007). Nessa direção, e com base na definição de Huber (1991), Alavi e Leidner (1999) definem o conhecimento como a crença pessoal justificada que incrementa a capacidade individual para realizar uma ação eficaz.

Complementarmente à visão de que o conhecimento é criado por indivíduos, a partir dos estudos de Polanyi (1966), Nonaka e Takeuchi (1997) distinguem o conhecimento em explícito e tácito. O conhecimento explícito é facilmente comunicado e compartilhado através da linguagem formal e sistemática. Pode ser expresso em palavras, números, manuais, entre outros. Já o conhecimento tácito, ao contrário do explícito, é pessoal, específico de um contexto, difícil de ser formulado e comunicado aos outros. Está relacionado aos modelos mentais (esquemas, paradigmas, crenças, pontos de vista) e know-how de cada indivíduo.

Visto que o conhecimento tácito é essencial para o entendimento do explícito, existe uma interação entre esses dois tipos de conhecimento (Uriarte, 2008). Segundo Nonaka e Takeuchi (1997) é essa interação que forma a espiral do conhecimento organizacional, representada por quatro etapas de conversão do conhecimento: a) socialização: compartilhamento do conhecimento tácito entre os indivíduos; b) externalização: explicitação do conhecimento tácito individual para o grupo; c) combinação: sistematização e propagação do conhecimento explícito gerado na etapa anterior; d) internalização: incorporação do conhecimento explícito pelo indivíduo, tornando-o tácito novamente.

A Gestão do Conhecimento refere-se aos processos sistemáticos e intencionais pelos quais a organização adquire, organiza e comunica conhecimento tácito e explícito dos colaboradores para que estes utilizem esse recurso de forma a serem mais eficazes e produtivos em suas funções (Alavi \& Leidner, 1999). Em consonância, Kianto e Andreeva (2014), afirmam que a GC visa maximizar a eficiência do recurso conhecimento e sua contribuição com os resultados da organização.

Nesta tentativa de gerenciar o conhecimento, os desafios maiores estão relacionados ao conhecimento tácito, pois por sua natureza, a identificação e explicitação deste tipo de conhecimento para a posterior integração aos bens e serviços, é uma tarefa complexa (Grant, 1996). Esta complexa atividade de explicitação de conhecimento tácito pode ser suportada por diversas metodologias, algumas delas relacionadas com a Engenharia do Conhecimento.

\subsection{Metodologias de Engenharia do Conhecimento}

A Engenharia do Conhecimento tem sua gênese na Inteligência Artificial e evoluiu, no final da década de 1970, como uma subárea dedicada à concepção, desenvolvimento e implantação de sistemas especialistas, 
sistemas baseados em conhecimento e sistemas intensivos em conhecimento (Studer, Benjamins, \& Fensel, 1998; Schreiber et al., 2000).

Os princípios que fundamentam a Engenharia do Conhecimento são: a) a EC não é uma espécie de "mineração da cabeça do especialista", mas aborda diferentes aspectos dos modelos humanos de conhecimento; b) a modelagem do conhecimento baseia-se em dois princípios: primeiro na estrutura conceitual e, depois, nos detalhes de programação do sistema baseado em conhecimento; c) um projeto de sistema baseado no conhecimento deve ser desenvolvido de forma controlada, em espiral, com uma metodologia que possibilite isso; e d) o conhecimento tem uma estrutura interna estável que pode ser distinguido em tipos específicos e papéis (Schreiber et al., 2000).

De acordo com um estudo realizado por Dias e Pacheco (2009), as principais metodologias usadas nas atividades de EC, são: CommonKADS; MAS-CommonKADS; Methodology for Knowledge-Based Engineering Applications (MOKA); Model-based and Incremental Knowledge Engineering (MIKE); IDEAL; SPEDE; eXtreme Programming of Knowledge Based Systems (XP.K); VITAL; On-To-Knowledge (OTK).

Para atingir o objetivo proposto neste estudo, escolheu-se a metodologia CommonKADS, criada por Schreiber et al. (2002). Esta metodologia está alinhada com os objetivos do presente estudo pelas seguintes razões: a) suporta a modelagem de elementos do contexto organizacional de forma abrangente e com profundidade; b) possibilita olhar a organização sob a perspectiva da orientação ao conhecimento; e c) descreve a situação atual e futura da organização, que permite planejar ações fundamentadas em melhorias organizacionais. A metodologia CommonKADS tem sido utilizada em estudos prévios em diversos contextos para modelagem organizacional considerando o recurso 'conhecimento' (Post et al., 1997; Titah et al., 2017).

\subsection{CommonKADS}

A metodologia CommonKADS originou-se da necessidade de construir sistemas de conhecimento com qualidade. É uma abordagem que permite a sistematização do conhecimento de forma estruturada, controlável e replicável da área de EC (Schreiber et al., 2002).

Além disso, três questões são fundamentais no processo de modelagem de um sistema de conhecimento utilizando-se a metodologia: Por quê? O quê? Como? Para responder a estas questões, a metodologia é organizada em três níveis de modelos (Schreiber et al., 2002):

a) Nível de contexto: tem por objetivo identificar os elementos fundamentais do ambiente e do contexto de um sistema de conhecimento a partir dos Modelos de Organização, Tarefa e Agente;

b) Nível conceitual: tem por objetivo explicitar e formalizar o conhecimento, bem como destacar de que modo ocorrem as interações entre os agentes envolvidos em sua utilização, por meio dos Modelos do Conhecimento e de Comunicação;

c) Nível de artefato: tem por objetivo apresentar todos os detalhamentos necessários para a construção de um Sistema de Conhecimento, utilizando-se do Modelo de Projeto para tal.

Os Modelos de Organização, Tarefa e Agente analisam o ambiente organizacional e os fatores críticos para o sucesso de um sistema de conhecimento. Os Modelos de Comunicação e Conhecimento produzem uma descrição conceitual das funções de resolução de problema dos dados, que são tratados e gerados por um sistema de conhecimento. Já o Modelo de Projeto converte os modelos citados anteriormente em uma especificação técnica que é a base para a implementação de um software. Schreiber et al. (2000) explica, ainda, que não é necessário utilizar todos os modelos, isso dependerá dos objetivos do projeto e das experiências adquiridas quando se executa o projeto. Desta forma, nesta pesquisa, foram utilizadas as planilhas do Modelo da Organização, que são explicadas com mais detalhes na seção de resultados.

\section{MÉTODO}

Esta seção tem o objetivo de apresentar a caracterização do universo de pesquisa; procedimentos metodológicos utilizados; e as considerações ético-legais pertinentes ao estudo. 


\subsection{Caracterização do universo de Pesquisa}

A pesquisa foi realizada em uma empresa da área da saúde que iniciou sua trajetória no ano de 2011, situada na cidade de Florianópolis. A clínica oferece serviços relacionados às especialidades de fisioterapia, massoterapia, naturologia e educação física. A equipe de trabalho é composta por duas recepcionistas e nove profissionais que compõe a equipe multidisciplinar (educadores físicos, fisioterapeutas, massoterapeuta, naturólogo, nutricionista), dentre eles o proprietário e administradora da clínica. Destes, todos se dispuseram a colaborar com a pesquisa, totalizando onze pessoas.

A escolha por esta clínica deve-se ao fato desta ter como prática a realização de um grupo de estudos, semanalmente, com duração de duas horas, em que os profissionais da equipe multidisciplinar estudam os casos clínicos dos pacientes atendidos no decorrer da semana. Neste espaço, ocorre o compartilhamento da experiência vivenciada no atendimento ao paciente e as peculiaridades de cada caso clínico. Ainda, os profissionais da equipe multidisciplinar discutem qual é a abordagem de tratamento ideal frente às necessidades apresentadas pelo paciente.

Entretanto, não ocorre o gerenciamento do conhecimento compartilhado no grupo de estudos, conforme mencionado pela administradora em uma reunião inicial a respeito da execução do estudo, carecendo, assim, de solução para esta questão. A escolha desta clínica se justifica, também, devido a acessibilidade oportunizada aos pesquisadores por parte da administração.

Salienta-se que o grupo de estudos faz parte do processo de atendimento ao paciente. Portanto, nesta pesquisa, é analisado o processo de atendimento ao paciente, com foco no grupo de estudos. Além disso, outro fator preponderante para analisar o processo completo, deve-se ao fato de que os diálogos que ocorrem no grupo de estudos têm relação direta com as demais etapas do processo e vice-versa.

\subsection{Procedimentos Metodológicos}

Esta pesquisa foi desenvolvida a partir de um estudo de caso, porque objetiva-se investigar empiricamente um acontecimento contemporâneo em que não é possível controlar os eventos comportamentais e não se tem clareza entre os limites do fenômeno e do contexto. Destaca-se, ainda, que este estudo de caso é do tipo descritivo, que tem o intuito de descrever um fenômeno atual em seu contexto de vida real (Yin, 2014). O fenômeno, neste estudo, conforme mencionado anteriormente, é o grupo de estudos realizado na organização pesquisada e a abordagem será qualitativa. A escolha por esta abordagem justifica-se por possibilitar que o caso seja estudado em profundidade, além de se mostrar importante para estudos de gestão (Patton, 2005).

Em estudos de caso qualitativos é recomendado que os dados sejam coletados combinando técnicas como observações, entrevistas, documentos etc. (Patton, 2005; Creswell, 2014). Os dados desta pesquisa foram coletados por meio de observação não participante e entrevistas semiestruturadas $A$ observação não participante foi escolhida porque os pesquisadores se abstiveram de intervenções e interrupções no fenômeno observado (Adler \& Adler, 1998). Já a entrevista semiestruturada, composta de perguntas previamente definidas, permitiu aos pesquisadores a exploração de questões complexas e esclarecimento de respostas quando necessário (Barriball \& While, 1994).

No quadro 1 expõem-se as três etapas do estudo. Em cada etapa se propôs atingir um objetivo específico desta pesquisa, explicitando o como (método de coleta de dados), com quem (os participantes) e os resultado obtidos. 
Quadro 1 - Síntese das etapas da pesquisa

\begin{tabular}{|c|c|c|c|c|}
\hline Etapa & Objetivo & Como? & Com quem? & Resultado \\
\hline \multirow[t]{2}{*}{1} & \multirow{2}{*}{$\begin{array}{c}\text { Caracterizar a } \\
\text { dinâmica do processo } \\
\text { de compartilhamento } \\
\text { de conhecimento }\end{array}$} & $\begin{array}{c}\text { Observação não } \\
\text { participante }\end{array}$ & $\begin{array}{l}\text { Recepcionistas e equipe } \\
\text { multidisciplinar } \\
\text { (11 pessoas) }\end{array}$ & \multirow{2}{*}{$\begin{array}{l}\text { CommonKADS: } \\
\text { Planilha OM-1 }\end{array}$} \\
\hline & & $\begin{array}{c}\text { Entrevista } \\
\text { semiestruturada }\end{array}$ & Administradora da clínica & \\
\hline 2 & $\begin{array}{l}\text { Mapear ativos de } \\
\text { conhecimento e } \\
\text { tarefas intensivas em } \\
\text { conhecimento }\end{array}$ & $\begin{array}{c}\text { Entrevista } \\
\text { semiestruturada }\end{array}$ & Administradora da clínica & $\begin{array}{l}\text { CommonKADS: } \\
\text { Planilha OM-2 } \\
\text { Planilha OM-3 } \\
\text { Planilha OM-4 }\end{array}$ \\
\hline 3 & $\begin{array}{l}\text { Analisar a viabilidade } \\
\text { da solução proposta }\end{array}$ & $\begin{array}{c}\text { Entrevista } \\
\text { semiestruturada }\end{array}$ & Administradora da clínica & $\begin{array}{l}\text { CommonKADS: } \\
\text { Planilha OM-5 }\end{array}$ \\
\hline
\end{tabular}

Fonte: elaborado pelos autores (2019).

Na primeira etapa o objetivo era caracterizar a dinâmica do processo de compartilhamento de conhecimento. Foram realizadas três observações, em três semanas consecutivas, e cada uma teve duração de duas horas. Todos os profissionais que se propuseram a participar desta pesquisa estavam presentes nestas observações. A importância de realizá-las em três semanas consecutivas, deu-se pelo fato de que alguns casos clínicos são levados ao grupo de estudos mais de uma vez, oportunizando à equipe multidisciplinar um aprofundamento no estudo do caso e acompanhamento da evolução clínica do paciente. Sob a forma de notas de campo, os dados coletados foram registrados em sua forma bruta e cronológica. No total, a pesquisadora presenciou o compartilhamento de 21 casos clínicos.

Posteriormente, para complementar os registros e identificar pontos críticos no processo de atendimento ao paciente, que não haviam sido esclarecidos durante as observações, realizou-se uma entrevista semiestruturada com a administradora da clínica e, também, fisioterapeuta. O resultado desta etapa é o preenchimento da planilha de problemas e oportunidades - OM-1 do CommonKADS.

No que tange ao segundo objetivo, o mapeamento dos ativos de conhecimento e tarefas intensivas em conhecimento foi realizado por meio de três entrevistas semiestruturadas, com a administradora da clínica. As entrevistas foram realizadas com base nos elementos da planilha de descrição da área foco do problema OM-2; planilha de detalhamento de processos - OM-3; e planilha de ativos de conhecimento - OM-4.

A análise de viabilidade da solução proposta (planilha OM-5) foi construída pelos pesquisadores, em conjunto com a administradora da clínica, por meio de uma entrevista semiestrutura, com o intuito de propor uma solução condizente com sua viabilidade de execução. Ressalta-se que todas as planilhas elaboradas foram apresentadas à administradora, que validou a consistência de cada uma delas, de acordo com o que foi abordado nas entrevistas.

Para que não haja redundância de informações, explica-se, na próxima seção, cada planilha CommonKADS utilizada e os resultados oriundos do preenchimento das mesmas, com o intuito de atender ao objetivo deste estudo.

\subsection{Considerações ético-legais}

A coleta de dados respeitou os procedimentos éticos relativos a pesquisas envolvendo seres humanos, de acordo com a Resolução no 196, de 10 de outubro de 1996 (http://conselho.saude.gov.br/resolucoes/reso_96.htm), e a Resolução no 466, de dezembro de 2012 (https://conselho.saude.gov.br/resolucoes/2012/Reso466.pdf), ambas do Conselho Nacional de Saúde (CNS). 
Neste sentido, a pesquisa foi iniciada após a aprovação do Comitê de Ética em Pesquisa envolvendo Seres Humanos (CEPSH), com o parecer no 1.871.386. Antes de iniciar as atividades desta pesquisa, apresentou-se o Termo de Consentimento Livre e Esclarecido, previamente aprovado pelo CEPSH, leu-se em conjunto com os participantes e, com a concordância de todos, coletou-se as assinaturas nos documentos, para que assim ficasse registrado. Além disso, é válido ressaltar que, durante todas as etapas da pesquisa, os clientes da clínica foram identificados por números, garantindo-lhes o anonimato.

\section{RESULTADOS}

Inicialmente foi preenchida a planilha de problemas e oportunidades - OM 1 (Quadro 2), que descreve o contexto organizacional da clínica, relacionando os problemas e oportunidades, assim como a solução correspondente. Descreve ainda os aspectos gerais, que são invariantes e necessários para entendimento do contexto organizacional.

Quadro 2 - Planilha de problemas e oportunidades

\begin{tabular}{|c|c|}
\hline $\begin{array}{c}\text { Modelo da } \\
\text { Organização }\end{array}$ & Planilha de problemas e oportunidades - OM-1 \\
\hline \multirow[b]{2}{*}{$\begin{array}{c}\text { Problemas e } \\
\text { Oportunidades }\end{array}$} & $\begin{array}{l}\text { Problemas: } \\
\text { - Conhecimento não explicitado } \\
\text { - Conhecimento sem possibilidade de reuso } \\
\text { - Memória organizacional não preservada }\end{array}$ \\
\hline & $\begin{array}{l}\text { Oportunidades: } \\
\text { - Crescimento intelectual dos profissionais } \\
\text { - Otimização dos processos internos } \\
\text { - Inovação nos serviços prestados } \\
\text { - Comunicação facilitada }\end{array}$ \\
\hline $\begin{array}{c}\text { Contexto } \\
\text { Organizacional }\end{array}$ & $\begin{array}{l}\text { Missão: Promover saúde através da ciência que vai além do diagnóstico e tratamento, } \\
\text { contribuindo para o desenvolvimento da plenitude física, através de um olhar sensível e } \\
\text { dedicado para a compreensão do ser humano como um todo, com as suas características } \\
\text { e objetivos ímpares, em favor de um corpo mais livre e espontâneo que possibilite a } \\
\text { expressão da verdadeira essência humana. } \\
\text { Observação: foi autorizado, para divulgação neste trabalho, apenas parte das informações } \\
\text { referentes ao contexto organizacional, devido ao sigilo de informações estratégicas } \\
\text { solicitado pela administradora da clínica. }\end{array}$ \\
\hline Soluções & $\begin{array}{l}\text { - Utilização de práticas de GC (storytelling, mentoring, comunidade de práticas etc.) e } \\
\text { agentes computacionais (plataformas colaborativas, e-mail, sistemas especialistas etc.) } \\
\text { para codificação, estruturação e armazenamento do conhecimento. }\end{array}$ \\
\hline
\end{tabular}

Fonte: elaborado pelos autores (2019) baseado em Schreiber et al. (2000).

A planilha de descrição da área foco do problema - OM-2 (Quadro 3) complementa a planilha anterior apresentando, de forma objetiva, os elementos que compõem o processo de atendimento ao paciente: pessoas envolvidas, recursos utilizados, os conhecimentos necessários e influência exercida pela relação de cultura e poder. 
Quadro 3 - Descrição da área foco do problema

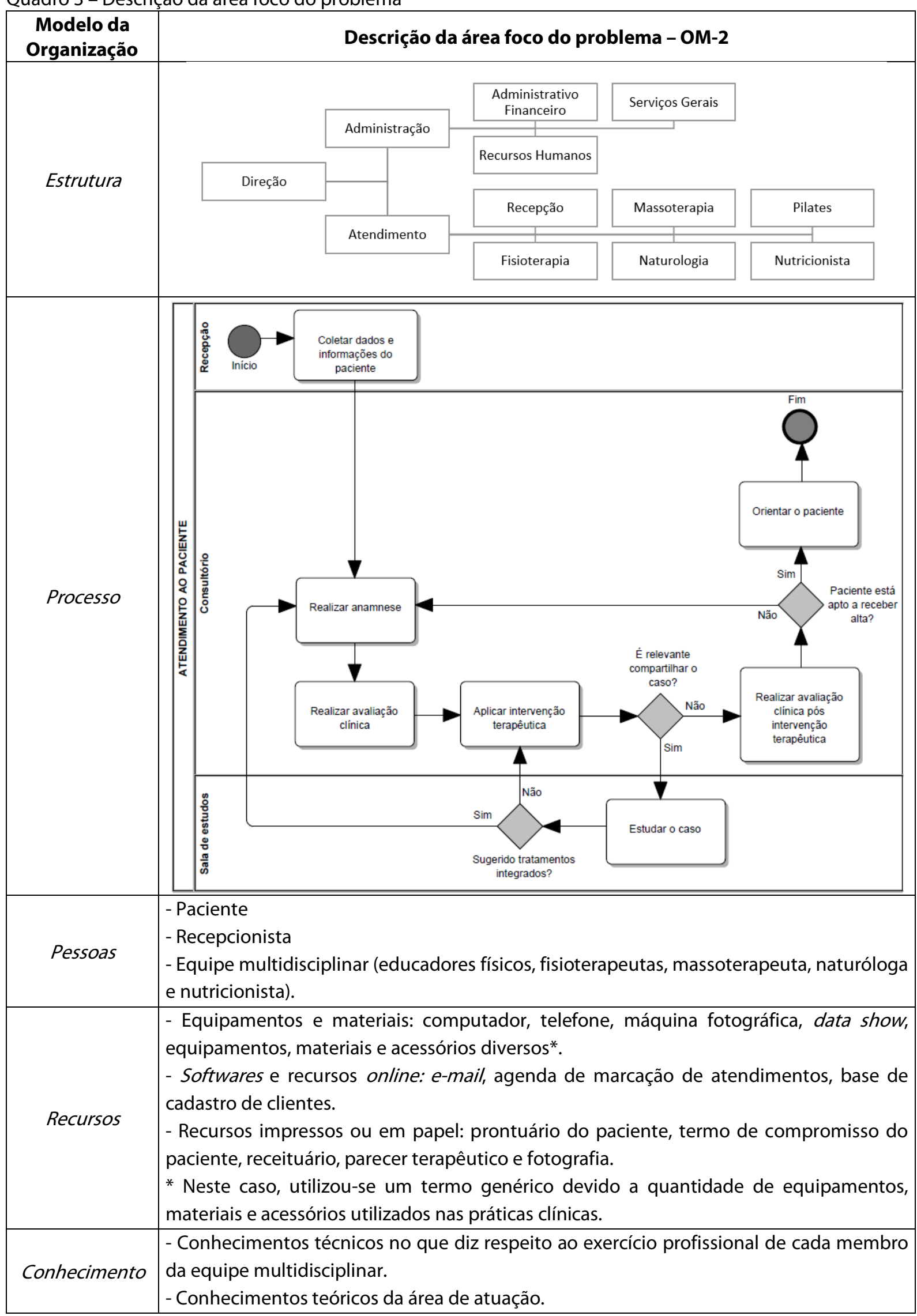




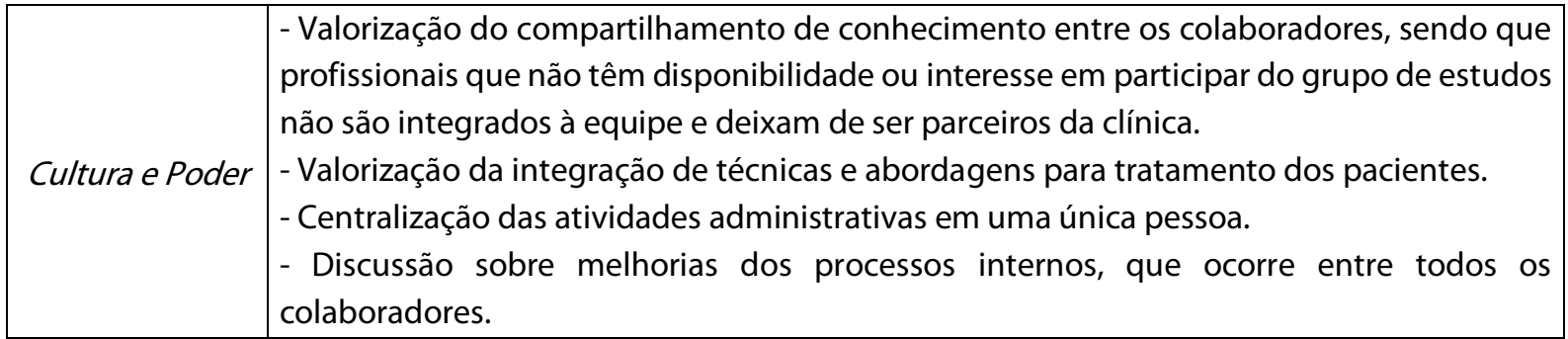

Fonte: elaborado pelos autores (2019) baseado em Schreiber et al. (2000).

Na terceira planilha - OM-3 (Quadro 4), as tarefas do processo de atendimento ao paciente, conforme identificado na planilha anterior, são descritas detalhadamente. Descreve-se as tarefas e as informações pertinentes a cada uma delas: realizador, local em que ocorre, ativos de conhecimento, se é intensiva em conhecimento e sua relevância no processo. A relevância foi classificada de 1 (um) a 5 (cinco), sendo 1 nada relevante e 5 muito relevante.

Quadro 4 - Planilha de detalhamento de processos

\begin{tabular}{|c|c|c|c|c|c|c|}
\hline & $\begin{array}{c}\text { Modelo da } \\
\text { Organização }\end{array}$ & & nilha de det & amento de proces & - OM-3 & \\
\hline$N^{o}$ & Tarefa & Realizada por & Onde & $\begin{array}{c}\text { Ativo de } \\
\text { Conhecimento }\end{array}$ & $\begin{array}{c}\text { Intensivo } \\
\text { Sim/Não }\end{array}$ & $\begin{array}{c}\text { Relevância } \\
\text { 1 a } 5\end{array}$ \\
\hline 01 & $\begin{array}{l}\text { Coletar dados e } \\
\text { informações do } \\
\text { paciente }\end{array}$ & Recepcionista & Recepção & Inexistente & Não & 4 \\
\hline 02 & Realizar anamnese & $\begin{array}{c}\text { Equipe } \\
\text { multidisciplinar }\end{array}$ & Consultório & Histórico pessoal & Sim & 5 \\
\hline 03 & $\begin{array}{c}\text { Realizar avaliação } \\
\text { clínica }\end{array}$ & $\begin{array}{c}\text { Equipe } \\
\text { multidisciplinar }\end{array}$ & Consultório & $\begin{array}{c}\text { Diagnóstico e } \\
\text { Indicação clínica }\end{array}$ & Sim & 5 \\
\hline 04 & $\begin{array}{c}\text { Aplicar } \\
\text { intervenção } \\
\text { terapêutica }\end{array}$ & $\begin{array}{c}\text { Equipe } \\
\text { multidisciplinar }\end{array}$ & Consultório & $\begin{array}{l}\text { Resposta à } \\
\text { intervenção } \\
\text { terapêutica }\end{array}$ & Sim & 5 \\
\hline 05 & Estudar o caso & $\begin{array}{c}\text { Equipe } \\
\text { multidisciplinar }\end{array}$ & $\begin{array}{c}\text { Sala de } \\
\text { Reuniões }\end{array}$ & $\begin{array}{l}\text { Considerações e } \\
\text { conclusões do } \\
\text { grupo de estudos }\end{array}$ & Sim & 5 \\
\hline 06 & $\begin{array}{c}\text { Realizar avaliação } \\
\text { clínica pós } \\
\text { intervenção } \\
\text { terapêutica }\end{array}$ & $\begin{array}{c}\text { Equipe } \\
\text { multidisciplinar }\end{array}$ & Consultório & $\begin{array}{l}\text { Resultados da } \\
\text { intervenção } \\
\text { terapêutica }\end{array}$ & Sim & 5 \\
\hline 07 & $\begin{array}{l}\text { Orientar o } \\
\text { paciente }\end{array}$ & $\begin{array}{c}\text { Equipe } \\
\text { multidisciplinar }\end{array}$ & Consultório & $\begin{array}{l}\text { Considerações e } \\
\text { conclusões da } \\
\text { intervenção } \\
\text { terapêutica }\end{array}$ & Sim & 5 \\
\hline
\end{tabular}

Fonte: elaborado pelos autores (2019) baseado em Schreiber et al. (2000).

Cabe ressaltar que a tarefa 01 não é intensiva em conhecimento, dada a natureza da tarefa, na qual coletam-se dados e informações. Pode ser realizada com um protocolo de atendimento, não requerendo conhecimentos específicos para realização desta.

A quarta planilha, denominada planilha de ativos de conhecimento - OM-4 (Quadro 5), lista os ativos de conhecimento identificados na planilha OM-3. A perspectiva, neste caso, é de que os "pedaços de conhecimento" são importantes como ativos e que se encontram em uso pelos trabalhadores da organização com o propósito de executar uma determinada tarefa ou processo. Ainda, identifica o agente possuidor e onde 
este conhecimento é utilizado. Também pontua se estão corretos a forma, lugar e tempo em que ocorre, assim como a qualidade deste ativo de conhecimento.

Quadro 5 - Planilha de ativos de conhecimento

\begin{tabular}{|c|c|c|c|c|c|c|}
\hline \multicolumn{2}{|c|}{ Modelo da Organização } & \multicolumn{5}{|c|}{ Ativos de conhecimento - OM-4 } \\
\hline $\begin{array}{c}\text { Ativo de } \\
\text { Conhecimento }\end{array}$ & Possuído por & Usado em & $\begin{array}{l}\text { Forma } \\
\text { correta? }\end{array}$ & $\begin{array}{l}\text { Lugar } \\
\text { correto? }\end{array}$ & $\begin{array}{l}\text { No tempo } \\
\text { correto? }\end{array}$ & $\begin{array}{l}\text { Qualidade } \\
\text { correta? }\end{array}$ \\
\hline Histórico pessoal & Paciente & $\begin{array}{c}\text { Fisioterapia, } \\
\text { Nutrição, } \\
\text { Naturologia, } \\
\text { Pilates e/ou } \\
\text { Massoterapia. }\end{array}$ & Sim & Sim & Sim & Sim \\
\hline $\begin{array}{c}\text { Diagnóstico e } \\
\text { indicação clínica }\end{array}$ & $\begin{array}{c}\text { Equipe } \\
\text { multidisciplinar }\end{array}$ & $\begin{array}{c}\text { Fisioterapia, } \\
\text { Nutrição, } \\
\text { Naturologia, } \\
\text { Pilates e/ou } \\
\text { Massoterapia. }\end{array}$ & Sim & Sim & Sim & Sim \\
\hline $\begin{array}{l}\text { Resposta à } \\
\text { intervenção } \\
\text { terapêutica }\end{array}$ & $\begin{array}{c}\text { Paciente e } \\
\text { equipe } \\
\text { multidisciplinar }\end{array}$ & $\begin{array}{c}\text { Fisioterapia, } \\
\text { Nutrição, } \\
\text { Naturologia, } \\
\text { Pilates e/ou } \\
\text { Massoterapia. }\end{array}$ & Sim & Sim & Sim & Sim \\
\hline $\begin{array}{l}\text { Considerações e } \\
\text { conclusões } \\
\text { do grupo de } \\
\text { estudos }\end{array}$ & $\begin{array}{c}\text { Equipe } \\
\text { multidisciplinar }\end{array}$ & $\begin{array}{c}\text { Fisioterapia, } \\
\text { Nutrição, } \\
\text { Naturologia, } \\
\text { Pilates e/ou } \\
\text { Massoterapia. }\end{array}$ & Não & Não & Não & Não \\
\hline $\begin{array}{l}\text { Resultados da } \\
\text { intervenção } \\
\text { terapêutica }\end{array}$ & $\begin{array}{c}\text { Paciente e } \\
\text { equipe } \\
\text { multidisciplinar }\end{array}$ & $\begin{array}{c}\text { Fisioterapia, } \\
\text { Nutrição, } \\
\text { Naturologia, } \\
\text { Pilates e/ou } \\
\text { Massoterapia. }\end{array}$ & Sim & Sim & Sim & Sim \\
\hline $\begin{array}{l}\text { Considerações e } \\
\text { conclusões da } \\
\text { intervenção } \\
\text { terapêutica }\end{array}$ & $\begin{array}{c}\text { Equipe } \\
\text { multidisciplinar }\end{array}$ & $\begin{array}{c}\text { Fisioterapia, } \\
\text { Nutrição, } \\
\text { Naturologia, } \\
\text { Pilates e/ou } \\
\text { Massoterapia. }\end{array}$ & Sim & Sim & Sim & Sim \\
\hline
\end{tabular}

Fonte: elaborado pelos autores (2019) baseado em Schreiber et al. (2000).

De acordo com as entrevistas realizadas para coleta de dados elucida-se que, no contexto estudado, o ativo de conhecimento 'histórico pessoal' refere-se às informações do paciente sobre suas afecções médicas anteriores e tratamentos realizados, aliando-os à visão integral do paciente, como por exemplo, a relação das afecções com seu estado emocional presente e passado, suas relações interpessoais, o ambiente em que vive e experiências vivenciadas.

$\mathrm{O}$ ativo de conhecimento 'diagnóstico e indicação clínica' compreende a determinação de uma doença ou estado físico e emocional do paciente, que possibilita a tomada de decisão para executar a intervenção terapêutica. Já a 'resposta à intervenção terapêutica' é um ativo de conhecimento que concerne ao resultado obtido com a intervenção terapêutica, no âmbito físco e emocional, com foco nas causas da 
doença. Nesta etapa, a administradora evidencia a importância de realizar um atendimento multidisciplinar ao paciente, dado que são conhecimentos de especialistas diferentes em prol de um objetivo comum.

$\mathrm{O}$ ativo de conhecimento 'considerações e conclusões do grupo de estudos' trata-se das inferências realizadas a partir do estudo do caso, conforme procedimento que ocorre no grupo de estudos explicado anteriormente. Destaca-se que ele é o único ativo que não está na forma, lugar, tempo e qualidade correta, porque falta formalização dos conhecimentos tácitos e individuais, específico do grupo de estudos, em conhecimentos explícitos, documentados e compartilhados com todos.

Os 'resultados da intervenção interapêutica' dizem respeito às percepções do paciente em relação ao resultado de seu tratamento e estado físico e emocional atual, bem como às percepções e conhecimentos dos profissionais da saúde que o trataram. Por fim, as 'considerações e conclusões da intervenção terapêutica' são os ativos de conhecimento, tácitos e explícitos, relacionados às orientações dadas ao paciente para que ele possa continuar cuidando de sua saúde.

Para finalizar o Modelo da Organização, verificou-se a viabilidade de uma solução proposta para os problemas identificados na planilha OM-1 (Quadro 2). Desta forma, o checklist de viabilidade (Quadro 6) foi construído considerando-se os seguintes itens: a) oportunidade mais promissora para aplicação da solução e qual é a melhor direção para esta; b) benefícios versus custos; c) quais tecnologias necessárias para a solução estão disponíveis e ao alcance; e d) outras ações do projeto que podem ser realizadas com sucesso.

Quadro 6 - Planilha de análise de viabilidade

\begin{tabular}{|c|c|}
\hline $\begin{array}{c}\text { Modelo da } \\
\text { Organização }\end{array}$ & Checklist de viabilidade - OM-5 \\
\hline $\begin{array}{l}\text { Viabilidade do } \\
\text { Negócio }\end{array}$ & $\begin{array}{l}\text { Com a possibilidade de retenção, compartilhamento e reuso do conhecimento de } \\
\text { forma sistematizada e estruturada, os benefícios esperados para a organização } \\
\text { referem-se à preservação da Memória organizacional e, consequente, melhoria na } \\
\text { qualidade dos serviços prestados, aprendizagem organizacional, oportunidade de } \\
\text { inovação e diferencial competitivo no setor em que atua. A execução do projeto exige } \\
\text { disponibilidade de tempo e esforço intelectual para que se obtenha sucesso. Sugere- } \\
\text { se a utilização de suporte tecnológico, como solução, especificamente uma plataforma } \\
\text { colaborativa, que possibilite a retenção, o compartilhamento e o reuso do } \\
\text { conhecimento. Serão necessárias mudanças organizacionais, pois o grupo de estudos } \\
\text { necessitará de uma adaptação quanto ao registro do conhecimento compartilhado e, } \\
\text { posteriormente, ao seu reuso, seja durante o grupo de estudos ou nas demais etapas } \\
\text { do processo de atendimento ao paciente. Os riscos relacionam-se ao desvio de } \\
\text { propósito da utilização da tecnologia proposta, como por exemplo, utilizá-la somente } \\
\text { para registro de informações e não de conhecimento, bem como indisponibilidade de } \\
\text { tempo e dedicação para manter a base de conhecimento atualizada. }\end{array}$ \\
\hline $\begin{array}{c}\text { Viabilidade } \\
\text { técnica }\end{array}$ & $\begin{array}{l}\text { A tarefa executada pelo sistema baseado em conhecimento é de média complexidade, } \\
\text { uma vez que precisa permitir a estruturação das informações e conhecimento a serem } \\
\text { armazenados, facilitando assim o posterior reuso desses registros, assegurando } \\
\text { praticidade em sua utilização. Os aspectos críticos envolvem disponibilidade de tempo } \\
\text { e dedicação por parte dos envolvidos no projeto, para que ele ocorra de forma a } \\
\text { solucionar o problema existente, e todos devem ter clareza quanto ao objetivo da } \\
\text { plataforma colaborativa. A indicação de qualidade, validade e desempenho } \\
\text { satisfatório do sistema de conhecimento está diretamente relacionada à capacidade } \\
\text { de preservar o conhecimento organizacional de maneira prática e ágil, sendo que esta } \\
\text { pode ser medida com a aplicação de questionários aos usuários da solução. A interface } \\
\text { deverá ser pensada e elaborada com foco no usuário final, sendo de fácil usabilidade } \\
\text { e o mais intuitiva possível. Não haverá interação da plataforma colaborativa com } \\
\text { outros sistemas ou ferramentas utilizadas pela clínica, pois não existem técnicas } \\
\text { disponíveis para interoperabilidade e integração entre eles. Por ser uma ferramenta }\end{array}$ \\
\hline
\end{tabular}




\begin{tabular}{|c|l|}
\hline \multirow{1}{*}{$\begin{array}{c}\text { Viabilidade do } \\
\text { projeto }\end{array}$} & $\begin{array}{l}\text { online e gratuita, existe a possibilidade de haver uma descontinuidade de utilização } \\
\text { da plataforma colaborativa, caso a mesma esteja indisponível. }\end{array}$ \\
\hline $\begin{array}{l}\text { Os interessados no projeto (administradora e colaboradores da clínica) reconhecem o } \\
\text { valor de gerenciar o conhecimento organizacional e os benefícios desta prática, por } \\
\text { isso demonstram-se motivados a colaborar com a execução do mesmo. As } \\
\text { expecificamente para o contexto organizacional existente. Os meios de comunicação } \\
\text { necessários estarão disponíveis durante o projeto, possibilitando a interação entre os } \\
\text { envolvidos sempre que necessário. O fato de as pessoas não se adaptarem ao uso da } \\
\text { solução ou utilizarem a mesma de forma equivocada, são fatores que necessitam de } \\
\text { atenção. }\end{array}$ & $\begin{array}{l}\text { 1. Foco: gerenciar o conhecimento organizacional. } \\
\text { 2. Solução-alvo: utilização de uma plataforma colaborativa como apoio à retenção, } \\
\text { compartilhamento e reuso do conhecimento. } \\
\text { 3. Resultados esperados: preservação da Memória organizacional. } \\
\text { 4. Ações necessárias: entendimento do contexto organizacional existente, } \\
\text { implantação de uma solução que atenda as necessidades apresentadas, avaliação da } \\
\text { solução implantada. } \\
\text { 5. Riscos: se não houver dedicação dos colaboradores da organização para utilizar a } \\
\text { base de conhecimento, bem como adaptar-se às mudanças necessárias no processo } \\
\text { de atendimento ao paciente, a plataforma não preservará a memória organizacional, } \\
\text { conforme planejado. }\end{array}$ \\
\hline propostas \\
\hline
\end{tabular}

Fonte: elaborado pelos autores (2019) baseado em Schreiber et al. (2000).

\section{DISCUSSÃO}

Os resultados obtidos, a partir da planilha OM-2, sinalizam que a administração da clínica preza por parceiros que possam e queiram participar do grupo de estudos. Desta forma, se entendemos que os valores do líder são incorporados na cultura organizacional no médio prazo, podemos afirmar que existe uma cultura organizacional que valoriza o compartilhamento do conhecimento na clínica e que esta é estimulada e promovida pela administradora de forma consciente e intencional desde o processo de recrutamento de parceiros. Em concordância com este resultado, o recrutamento baseado em conhecimento tem sido visto em estudos prévios como um dos principais determinantes do sucesso dos processos de compartilhamento de conhecimento e inovação nas organizações (Inkinen, 2015; Davila, Andreeva, Varvakis, 2019). De forma complementar, a literatura existente também enfatiza que a cultura organizacional tem uma forte influência na Gestão do Conhecimento e na eficácia do compartilhamento do conhecimento (Yeh, Lai, \& Ho, 2006; Chang \& Lin, 2015). Essa evidência também concorda com o estudo de Anantatmula (2008) que verificou que a importância do líder está relacionada ao fomento de uma cultura e ambiente conveniente ao diálogo e compartilhamento do conhecimento espontâneo entre os membros da organização. Ainda, o líder é citado como exemplo, no sentido de influenciar as ações dos integrantes da organização, por isso a relevância dele na coordenação, colaboração e incentivo à prática de compartilhamento do conhecimento (Søndergaard, Kerr, \& Clegg, 2007; Anantatmula \& Kanungo, 2010). Na mesma linha, há o estudo de Wong e Aspinwall (2005), que menciona que o líder deve se dedicar à promoção de uma mentalidade organizacional, voltada à cooperação e compartilhamento do conhecimento e a construção de um ambiente no qual a criação de conhecimento e a aprendizagem possam acontecer, bem como apoiar e se comprometer continuamente com a GC.

Outro achado desta pesquisa diz respeito ao grupo de estudos, enquanto espaço de interação entre os indivíduos, que está intrinsecamente ligado à criação do conhecimento, uma prática que ocorre em espaços físicos de trabalho colaborativo por meio de interações face a face (Asian Productivity Organization [APO], 
2010). De acordo com Nonaka e Takeuchi (1997) a criação do conhecimento está associada às etapas de socialização, externalização, combinação e internalização.

Neste sentido, verificou-se que a etapa de socialização acontece na organização estudada, pois o conhecimento tácito é compartilhado entre os profissionais por meio da linguagem que está integrada às emoções e contextos específicos, conforme proposto por Nonaka e Takeuchi (1997). Ademais, o conhecimento tácito, no contexto das organizações de saúde, consiste nas experiências, percepções e julgamentos pessoais dos profissionais de saúde, que são desenvolvidos por meio da interação com os pacientes (Zhou \& Nunes, 2016), o que também apoia os achados deste estudo.

Ressalta-se que a socialização acontece periodicamente na clínica promovendo um fluxo contínuo de compartilhamento de conhecimento e ampliando a criação de conhecimento organizacional. Isto atesta a pesquisa de Dorow, Trzeciak, \& Rados (2018), realizada no setor da saúde, que afirma que o processo de compartilhamento do conhecimento não é um processo com início e fim, mas um processo contínuo que contribui com discussões relevantes entre os profissionais e prestação de um serviço com qualidade.

Já a etapa de externalização não acontece adequadamente, conforme verificado a partir do detalhamento do processo realizado na planilha $\mathrm{OM}-3$, que serviu de insumo para a planilha seguinte. Com os resultados obtidos com a planilha OM-4, infere-se que o ativo de conhecimento 'considerações e conclusões do grupo de estudos' não está na forma, lugar, tempo e qualidade correta, tendo em vista que os conhecimentos tácitos e individuais do grupo de estudos não são explicitados em documentos e compartilhados com todos. Esta etapa é fundamental para as organizações, uma vez que o conhecimento explicitado é base para reflexão e ação consciente (Nonaka \& von Krogh, 2009).

Ainda, a forma como cada ativo de conhecimento é entendida na organização pesquisada, a qual foca em uma perspectiva holística do ser humano, considerando-se o tratamento e cura física e emocional, ressalta a importância do compartilhamento do conhecimento na área da saúde. Isto confirma os estudos realizados em organizações de saúde em países emergentes (Assem \& Pabbi, 2016; Dorow, Trzeciak, \& Rados, 2018) e desenvolvidos (Zhou \& Nunes, 2016).

Além disso, a estratégia adotada na clínica, de atendimento e estudo multidisciplinar dos casos clínicos, permite que o paciente seja atendido com mais qualidade e eficácia, uma vez que diferentes conhecimentos, habilidades e visões de mundo são integradas, conforme mencionado pela administradora nas entrevistas. O estudo de Körner et al. (2016) confirma que o trabalho em equipe nas organizações de saúde é um dos critérios de qualidade mais importantes, pois a integração do conhecimento proporciona uma visão holística do paciente, bem como melhoria no desempenho da equipe.

Por conseguinte, as próximas duas etapas do processo de criação do conhecimento também não acontecem na clínica pesquisada. São elas: i) combinação, compreendida pelo processo de sistematização de conjuntos diferentes de conhecimentos explícitos em um sistema de conhecimento; e ii) internalização, em que o indivíduo aprende fazendo e incorpora o conhecimento explícito aos seus conhecimentos, tornando-o tácito novamente.

Portanto, de acordo as soluções propostas na planilha OM-1, sugere-se a utilização de agentes computacionais (plataformas colaborativas, e-mail, sistemas especialistas etc.). A viabilidade desta proposta de solução é demonstrada na planilha OM-5. Assim, as três últimas etapas de criação do conhecimento poderão ocorrer de forma adequada na clínica. Esta sugestão considera a flexibilidade da tecnologia da informação, bem como seu uso online, não limitando-se a espaço e tempo.

As tecnologias oferecem um espaço de interação e comunicação em grupo, que permite que os indivíduos tenham novas percepções e/ou interpretações mais precisas se comparado à perspectiva individual, o que resulta em novos conhecimentos individuais (Alavi \& Leidner, 2001), bem como otimização do processo de criação de conhecimento (Nonaka, 1994). Além disso, influenciam no processo de GC como um todo, pois permitem a aquisição, retenção e reutilização do conhecimento de forma sistematizada e estruturada, formando a memória organizacional (Stein, 1995; Wijnhoven, 1998). Em consonância com esta proposta, estudos anteriores demonstram que as tecnologias auxiliam e mediam o compartilhamento do conhecimento em rede entre profissionais da saúde (Whiddett, Tretiakov, \& Hunter, 2012). 


\section{CONSIDERAÇõES FINAIS}

Reiterando a importância do conhecimento como um recurso para as organizações intensivas em conhecimento, o presente artigo teve o propósito de compreender como os ativos de conhecimento estão inseridos no processo de atendimento ao paciente em uma organização do setor da saúde.

Os resultados deste artigo contribuem com a KBV, pois ajudam a entender, de forma sistemática, como o recurso conhecimento é gerenciado ao longo dos processos da saúde em um país emergente como o Brasil. Com os resultados obtidos por meio de observações, entrevistas e utilização da metodologia CommonKADS, infere-se que dos seis ativos de conhecimento identificados, o ativo de conhecimento referente ao grupo de estudos não está na forma, lugar, tempo e qualidade corretos. Isto influencia diretamente no processo de criação do conhecimento, que fica prejudicado, uma vez que apenas a socialização acontece de forma adequada, momento em que os profissionais compartilham conhecimento tácito. Já a etapa de externalização não ocorre, pois o conhecimento tácito não é formalizado e, por consequência, as etapas seguintes, combinação e internalização, também não ocorrem.

Desta forma, sugeriu-se a utilização de agentes computacionais para que as três últimas etapas de criação do conhecimento possam acontecer na clínica, sendo que a viabilidade desta proposta foi demonstrada no estudo. As tecnologias influenciam positivamente tanto a criação do conhecimento quanto o processo de GC e a preservação da memória organizacional.

Ademais, os resultados demonstraram que a cultura organizacional, orientada pelo líder, favorece o compartilhamento do conhecimento na organização estudada, bem como a prestação de um atendimento de qualidade aos pacientes. Portanto, é relevante que o líder disponha de tempo e espaço para esta prática, colabore e participe destes momentos em conjunto com os colaboradores da organização.

Ressalta-se, também, que a metodologia CommonKADS foi fundamental para o atingir o objetivo proposto neste estudo, uma vez que possibilitou obter uma visão geral do contexto organizacional; mapear e caracterizar o conhecimento envolvido nos processos-chave da organização; e analisar a viabilidade de uma solução orientada ao conhecimento. Assim, apesar dos resultados não poderem ser generalizados, para a empresa estudada, gerou subsídios para refletir e planejar ações futuras de forma a maximizar o uso e a operacionalização do conhecimento, enquanto recurso essencial para prestar um atendimento de qualidade aos pacientes.

Tais constatações contribuem com as pesquisas empíricas relacionadas à GC em organizações de saúde de países emergentes, pesquisa a solução para um problema de gerenciamento do conhecimento e exemplifica a aplicação do CommonKADS.

Como todo estudo, o presente artigo abre caminhos para novas pesquisas. Em primeiro lugar, o estudo apresentado analisou um caso específico do setor saúde. Outros setores intensivos em conhecimento poderiam ser estudados em busca de novas proposições visando identificar e confirmar as semelhanças e particularidades no que tange à Gestão do Conhecimento em diversos setores econômicos. Segundo, foi usado um método de $\mathrm{EC}$, como o CommonKADS, que permite uma caracterização estática do conhecimento. Sugerimos que futuras pesquisas considerem métodos que permitam analisar os aspectos dinâmicos da criação do conhecimento.

\section{REFERÊNCIAS}

Adler, P. A., \& Adler, P. (1994). Observational techniques. In N. K. Denzin \& Y. S. Lincoln (Eds.), Handbook of Qualitative Research (pp. 377-392). Thousand Oaks: Sage Publications, Inc.

Alavi, M., \& Leidner, D. (1999). Knowledge management systems: issues, challenges, and benefits. Communications of the Association for Information Systems, 1(1), 7.

Alavi, M., \& Leidner, D. E. (2001). Knowledge management and knowledge management systems: Conceptual foundations and research issues. MIS Quarterly, 107-136.

Alvesson, M. (2004). Knowledge work and knowledge-intensive firms. New York: Oxford University Press. 
Anantatmula, V. S. (2008). Leadership role in making effective use of KM. Vine, 38(4), 445-460.

Anantatmula, V. S., \& Kanungo, S. (2010). Modeling enablers for successful KM implementation. Journal of Knowledge Management, 14(1), 100-113.

Assem, P. B., \& Pabbi, K. A. (2016). Knowledge sharing among healthcare professionals in Ghana. VINE Journal of Information and Knowledge Management Systems, 46(4), 479-491.

Asian Productivity Organization (APO). (2010). Knowledge Management Tools and Techniques Manual. Tokyo: APO.

Barriball, K. L., \& While, A. (1994). Collecting data using a semi-structured interview: a discussion paper. Journal of Advanced Nursing-Institutional Subscription, 19(2), 328-335.

Cegarra-Navarro, J. G., \& Cepeda-Carrión, G. (2010). How to implement a knowledge management program in hospital-in-the-home units. Leadership in Health Services, 23(1), 46-56.

Cicone, P. A., Costa, C. K. F., Massuda, E. M., Vermelho, S. C. S. D., \& Gimenes, R. M. T. (2015). Gestão do Conhecimento em Organizações de Saúde: Revisão Sistemática de Literatura. Saúde e Pesquisa, 8(2), 379388.

Chang, C. L. H., \& Lin, T. C. (2015). The role of organizational culture in the knowledge management process. Journal of Knowledge Management, 19(3), 433-455.

Creswell, J. W. (2014). Investigação Qualitativa e Projeto de Pesquisa: escolhendo entre cinco abordagens (3. ed.). Porto Alegre: Penso.

Cruz, S. G., \& Ferreira, M. M. F. (2016). Gestão do conhecimento em instituições de saúde portuguesas. Revista Brasileira de Enfermagem, 69(3), 492-499.

Davenport, T., \& Prusak, L. (1998). Conhecimento Empresarial: como as organizações gerenciam seu capital intelectual. Rio de Janeiro: Campus.

Davila, G., Varvakis, G. \& North, K. (2019). Influência da Gestão Estratégica do Conhecimento na Inovação e no Desempenho da Empresa. Brazilian Business Review, 16 (3), 239-254.

Davila, G., Andreeva, T., \& Varvakis, G. (2019). Knowledge Management in Brazil: What Governance Mechanisms are Needed to Boost Innovation?. Management and Organization Review, 1-30.

Dias, M. M., \& Pacheco, R. C. D. S. (2009). Uma visão geral de metodologias para desenvolvimento de sistemas baseados em conhecimento. Revista de Ciência da Informação, 10(5).

Dorow, P. F., Trzeciak, D. S., \& Rados, G. J. V. (2018). Motivadores ao Compartilhamento de Conhecimento Tácito em Organizações Intensivas em Conhecimento. Revista Eletrônica de Ciência Administrativa, 17(3), 373-394.

El Morr, C., \& Subercaze, J. (2010). Knowledge management in healthcare. In M. M. C., Cunha, A. J., Tavares, \& R. Simoes (Orgs.), Handbook of research on developments in E-health and telemedicine: technological and social perspectives (pp. 490-510). USA: IGI Global.

Gonzalez, R. V. D., \& Martins, M. F. (2017). Knowledge Management Process: a theoretical-conceptual research. Gestão \& Produção, 24(2), 248-265.

Grant, R. M. (1996). Toward a knowledge-based theory of the firm. Strategic Management Journal, 17(S2), 109-122.

Huber, G. P. (1991). Organizational learning: The contributing processes and the literatures. Organization Science, 2(1), 88-115.

Inkinen, H. T., Kianto, A., \& Vanhala, M. (2015). Knowledge management practices and innovation performance in Finland. Baltic Journal of Management, 10(4), 432-455. 
Kabir, M. N. (2019). Knowledge-Based Social Entrepreneurship: Understanding Knowledge Economy, Innovation, and the Future of Social Entrepreneurship. New York: Springer.

Kaplan, R. S., \& Norton, D. P. (2004). Mapas estratégicos: convertendo ativos intangíveis em resultados tangíveis (7. ed.). Rio de Janeiro: Elsevier.

Kianto, A., \& Andreeva, T. (2014). Knowledge management practices and results in service oriented versus product-oriented companies. Knowledge and Process Management, 21(4), 221-230.

Körner, M., Lippenberger, C., Becker, S., Reichler, L., Müller, C., Zimmermann, L., ... \& Baumeister, H. (2016). Knowledge integration, teamwork and performance in health care. Journal of health organization and management, 30(2), 227-243.

Liu, F. C., Cheng, K. L., Chao, M., \& Tseng, H. M. (2012). Team innovation climate and knowledge sharing among healthcare managers: mediating effects of altruistic intentions. Chang Gung Medical Journal, 35(5), 408-419.

Nonaka, I. (1994). A dynamic theory of organizational knowledge creation. Organization Science, 5(1), 14-37.

Nonaka, I., \& Takeuchi, H. (1997). Criação de conhecimento na empresa (2. ed.). Rio de Janeiro: Campus.

Nonaka, I., \& von Krogh, G. (2009). Tacit knowledge and knowledge conversion: Controversy and advancement in organizational knowledge creation theory. Organization Science, 20(3), 635-652.

North, K., \& Kumta, G. (2018). Knowledge management: Value creation through organizational learning (2. ed.). Switzerlan: Springer.

Patton, M. Q. (2005) Qualitative Research. In B. S. Everitt \& D. C. Howell (Eds.), Encyclopedia of Statistics in Behavioral Science (Vol. 3, pp. 1633-1636). Chichester: John Wiley \& Sons, Inc.

Polanyi, M. (1966). The tacit dimension. London: Routledge and Kegan.

Post, W., Wielinga, B., De Hoog, R., \& Schreiber, G. (1997). Organizational modeling in commonkads: The emergency medical service. IEEE Expert, 12(6), 46-52.

Rocha, E. S. B., Nagliate, P., Furlan, C. E. B., Rocha Jr, K., Trevizan, M. A., \& Mendes, I. A. C. (2012). Gestão do conhecimento na saúde: revisão sistemática de literatura. Revista Latino-Americana de Enfermagem, 20(2), 392-400.

Schreiber, A. T., Akkermans, H., Anjewierden, A., Hoog, R., Shadbolt, N., Van de Velde, W., \& Wielinga, B. (2000). Knowledge engineering and management: the CommonKADS methodology. Massachusetts: MIT press.

Søndergaard, S., Kerr, M., \& Clegg, C. (2007). Sharing knowledge: contextualising socio-technical thinking and practice. The Learning Organization, 14(5), 423-435.

Starbuck, W. H. (1992). Learning by knowledge intensive firms. Journal of Management Studies, 29 (6), 713 740.

Stein, E. W. (1995). Organization memory: Review of concepts and recommendations for management. International Journal of Information Management, 15(1), 17-32.

Studer, R., Benjamins, V. R., \& Fensel, D. (1998). Knowledge engineering: principles and methods. Data \& Knowledge Engineering, 25(1-2), 161-197.

Titah, M., Aitouche, S., Mouss, M. D., \& Soussa, A. (2017). Externalising and reusing of tacit knowledge in manufacturing task. International Journal of Knowledge Management Studies, 8(3-4), 351-374.

Uriarte, F. A. (2008). Introduction to knowledge management. Indonesia: ASEAN Foundation. 
Whiddett, D., Tretiakov, A., \& Hunter, I. (2012). The use of information technologies for knowledge sharing by secondary healthcare organisations in New Zealand. International journal of medical informatics, 81(7), 500-506.

Wijnhoven, F. (1998). Designing organizational memories: Concept and method. Journal of Organizational Computing and Electronic Commerce, 8(1), 29-55.

Wong, K. Y., \& Aspinwall, E. (2005). An empirical study of the important factors for knowledge-management adoption in the SME sector. Journal of Knowledge Management, 9(3), 64-82.

Yeh, Y. J., Lai, S. Q., \& Ho, C. T. (2006). Knowledge management enablers: a case study. Industrial Management \& Data Systems, 106(6), 793-810.

Yin, R. K. (2014). Case study research: design and methods. (5. ed.) Thousand Oaks: SAGE Publications.

Zhou, L., \& Nunes, M. B. (2016). Barriers to knowledge sharing in Chinese healthcare referral services: an emergent theoretical model. Global health action, 9(1), 29964.

Zins, C. (2007). Conceptual approaches for defining data, information, and knowledge. Journal of the American Society for Information Science and Technology, 58(4), 479-493. 